\title{
microRNA-96 regulates the proliferation of nucleus pulposus cells by targeting ARID2/AKT signaling
}

\author{
BO TAO $^{1 *}$, JIAOYU Y $^{2 *}$, CHANGZHAO HUANG ${ }^{1}$, WANG XU $^{1}, \mathrm{CHAO}^{2}$ QIN $^{1}$, \\ LEI CHEN $^{1}$, JINCAI CHEN ${ }^{3}$, YAN GAO ${ }^{1}$ and RUIYING WANG ${ }^{1}$
}

\author{
${ }^{1}$ Department of Orthopedic Surgery, Affiliated Hospital of Guilin Medical University, Guilin, Guangxi 541001; \\ ${ }^{2}$ Department of Emergency Medicine, Fudan University Shanghai Cancer Center, Shanghai 200032; ${ }^{3}$ Department of \\ Orthopedic Surgery, The First Affiliated Hospital of Gannan Medical University, Ganzhou, Jiangxi 341000, P.R. China
}

Received September 29, 2016; Accepted June 2, 2017

DOI: $10.3892 / \mathrm{mmr} .2017 .7560$

\begin{abstract}
The aberrant proliferation of nucleus pulposus (NP) cells has been reported to be implicated in the pathogenesis of intervertebral disc degeneration (IDD). Previous studies have demonstrated that microRNAs (miRNAs), which are a group of small noncoding RNAs, are critical regulators of cell proliferation in various pathologies. However, the role of miRNA-96 (miR-96) in the proliferation of NP cells remains to be determined. In the present study, reverse transcription-quantitative polymerase chain reaction was used to investigate the expression of miR-96 in NP tissues from patients with IDD and healthy tissues from patients with traumatic lumbar fracture as the control. A dual-luciferase reporter assay was used to investigate whether AT-rich interaction domain 2 (ARID2) may be a direct target gene for miR-96. Furthermore, isolated NP cells from patients with IDD were transfected with miR-96 mimics and ARID2-targeting small interfering RNAs; cell proliferation, and the protein expression of Akt, phosphorylated Akt and ARID2 were examined, whereas the effects of an Akt inhibitor on NP cell proliferation were also evaluated. The present results demonstrated that miR-96 expression was significantly upregulated in IDD samples, and the level of miR-96 expression was positively associated with disc degeneration grade, which was evaluated by a modified Pfirrmann grading system. In addition, the current study identified ARID2 as a direct gene target of miR-96. Furthermore, it was demonstrated that ARID2 mRNA expression was inversely correlated with the expression of miR-96 in NP tissues. In addition, miR-96 overexpression promoted
\end{abstract}

Correspondence to: Professor Ruiying Wang, Department of Orthopedic Surgery, Affiliated Hospital of Guilin Medical University, 15 Lequn Road, Guilin, Guangxi 541001, P.R. China E-mail: rywang@glmc.edu.cn

${ }^{*}$ Contributed equally

Key words: AT-rich interaction domain 2, microRNA-96, nucleus pulposus cells, intervertebral disc degeneration
NP cell proliferation and induced Akt phosphorylation, which led to increased cyclin D1 translation. Notably, overexpression of ARID2 or treatment with an Akt inhibitor decreased the effect of miR-96 on NP cell proliferation. In conclusion, the results of the present study indicate that miR-96 may promote the proliferation of human degenerated NP cells by targeting ARID2 via activation of the Akt pathway, and potentially serves as a therapeutic target for IDD.

\section{Introduction}

Lower back pain is one the most common musculoskeletal disorders, inflicting a large economic burden on the healthcare system worldwide (1-3). The causes of lower back pain are complex, and intervertebral disc degeneration (IDD) is considered to contribute substantially to the development of this pain $(4,5)$. Although various etiological factors have been suggested to influence the development of IDD, including aging, genetic predisposition and environmental factors $(6,7)$, the underlying cellular mechanisms remain largely unknown. Several previous reports have demonstrated that the formation of clusters of nucleus pulposus (NP) cells and fibrocartilaginous tissue proliferation have important roles in IDD (8-10). However, the precise cause of enhanced proliferation of NP cells in IDD remains unclear.

MicroRNAs (miRNAs) are a category of small ( $\sim 22$ nucleotides), single-stranded, noncoding RNAs that exert their biological functions through recognition and binding to specific sequences within the 3'-untranslated region (3'-UTR) of target genes. miRNAs binding to these regions in mRNA leads to mRNA degradation or represses protein translation $(11,12)$. There is increasing evidence that indicates that miRNAs have important functions in various cellular processes, including cell proliferation, differentiation and apoptosis (13-15). In addition, a number of studies have demonstrated that dysregulated miRNAs participate in various human cancers and act as oncogenes or suppressor genes, depending the function of their targets $(16,17)$. Additionally, several miRNAs were reported to regulate various target genes, pathways and processes essential for the pathogenesis of IDD (18-20). However, although miRNAs have been investigated extensively in recent years, their roles in IDD development, and as potential markers for 
diagnosis and prognosis, remain unclear. As an important miRNA, miRNA-96 (miR-96) is involved in regulating the proliferation of various tumor types, including urothelial carcinoma, bladder cancer and colorectal cancer (21-23). As miR-96 is a crucial regulator of cell proliferation in various pathologies, and IDD is characterized by the abnormal proliferation of NP cells, we hypothesized that miR-96 may be involved in the development of IDD. Therefore, the aim of the present study was to evaluate the functional role of miR-96 in IDD and to investigate the underlying molecular mechanism.

\section{Materials and methods}

Ethics statement. All experimental protocols were approved by the Clinical Research Ethics Committee of The Affiliated Hospital of Guilin Medical University (Guilin, China). Written informed consent was obtained from all patients.

Patients and samples. NP tissue samples from patients with IDD or traumatic lumbar fracture were collected from patients undergoing discectomy in the Department of Orthopedics of the Affiliated Hospital of Guilin Medical University between June 2015 and June 2016. A total of 30 patients (mean age, $50 \pm 11.7$ years; age range, 40-63 years; 16 males, 14 females) with IDD, including patients with lumbar (L)4/5 ( $\mathrm{n}=22)$ and L5/sacral $1(n=8)$ disk herniations, and 5 patients with a recent traumatic lumbar fracture (mean age, 21.6 \pm 3.8 years; age range, 18-26 years; 3 males, 2 females) were enrolled in the present study. Patients with infections, previous lumbar disc surgery or idiopathic scoliosis were excluded. Routine magnetic resonance imaging scans of the lumbar spine were taken of each patient prior to surgery. The degree of disc degeneration was classified using a modified Pfirrmann grading system (24).

Isolation and culture of human NP cells. NP cells were isolated as described previously (25). NP tissue specimens were first washed twice with PBS. NP tissue was separated from annulus fibrosus tissue by visualizing under a stereotaxic microscope and was cut into pieces $\left(2-3 \mathrm{~mm}^{3}\right)$. NP cells were released from NP tissues by incubation with $0.25 \mathrm{mg} / \mathrm{ml}$ type II collagenase (Invitrogen; Thermo Fisher Scientific, Inc., Waltham, MA, USA) for $12 \mathrm{~h}$ at $37^{\circ} \mathrm{C}$ in Dulbecco's modified Eagle's medium (DMEM; Gibco; Thermo Fisher Scientific, Inc.). Following isolation, NP cells were resuspended in DMEM containing $10 \%$ fetal bovine serum (FBS; Gibco; Thermo Fisher Scientific, Inc.), $100 \mu \mathrm{g} / \mathrm{ml}$ streptomycin, $100 \mathrm{U} / \mathrm{ml}$ penicillin and $1 \%$ L-glutamine, and incubated at $37^{\circ} \mathrm{C}$ in a humidified atmosphere with $95 \%$ air and $5 \% \mathrm{CO}_{2}$. Fully confluent cells were detached by trypsinization, seeded into $35-\mathrm{mm}$ tissue culture dishes in complete culture medium (DMEM supplemented with $10 \% \mathrm{FBS}, 100 \mu \mathrm{g} / \mathrm{ml}$ streptomycin and $100 \mathrm{U} / \mathrm{ml}$ penicillin) and incubated at $37^{\circ} \mathrm{C}$ in a humidified atmosphere with $95 \%$ air and $5 \% \mathrm{CO}_{2}$. The medium was changed every 3 days. Second passage cells were used for experiments.

Oligonucleotides, constructs and transfections. A total of $24 \mathrm{~h}$ prior to transfection, cells were seeded into 6-well dishes in $2 \mathrm{ml} \mathrm{DMEM} \mathrm{containing} 1 \%$ penicillin/streptomycin. The medium used was discarded after $24 \mathrm{~h}$ and cells were washed twice with Opti-MEM I medium (Thermo Fisher Scientific, Inc.). Opti-MEM (11.5 ml) was added to each well. miR-96 mimic, miR-96 control, miR-96 inhibitor and miR-96 control inhibitor (5 $\mu$ l; Guangzhou RiboBio Co., Ltd., Guangzhou, China) were diluted in Opti-MEM I $(250 \mu \mathrm{l})$. The sequences of the oligonucleotides used were as follows: miR-96 mimic, forward 5'-CAGUAGGAAUAGACUUUCG-3', reverse 5'-GUCAUCCUUAUCUGA AAGC-3'; miR-96 control, forward 5'-UAAAGUGCUUAUAGUGCAGGUAG-3', reverse 5'-CUACCUGCACUAUAAGCACUUUA-3'; miR-96 inhibitor, forward 5'-AGCUAUAGACAAAAGCACG-3', reverse 5'-GUTCTGGCGCACGAAAGC-3'; and miR-96 inhibitor control, forward 5'-CAGUAGCUUAUACAGUAG-3' and reverse 5'-GCAGTACACACUUUATTGCT-3'. AT-rich interaction domain 2 (ARID2) plasmid, control siRNA and ARID2 small interfering (si)RNA were purchased from Guangzhou RiboBio Co., Ltd. The sequences of the siRNAs that were used were as follows: Control siRNA, forward 5'-GCTTGA GGGTCTGAATCTTGCT-3', reverse 5'-GTCCGCAGTCTT ACGAGGAG-3'; siARID2, forward 5'-CCAGCTCGAGGG ATTCAGGAATTGCTCCACCA-3', reverse 5'-CCAGGC GGCCGCCTCCTCTGGCAGTAATGGTCCT-3'. Cells were seeded at a density of $5 \times 10^{6}$ cells/well $16 \mathrm{~h}$ prior to transfection in DMEM with $1 \%$ penicillin/streptomycin. Briefly, $10 \mathrm{nM}$ miRNAs, $100 \mathrm{nM}$ siRNAs, $800 \mathrm{ng}$ ARID2 plasmid, $800 \mathrm{ng}$ control vector and were diluted in the serum-free medium at room temperature for $5 \mathrm{~min}$ using Lipofectamine 2000 as the transfection reagent (Thermo Fischer Scientific, Inc.). Then, the mixture was added to the cells (500 $\mu \mathrm{l} /$ well) and incubated in $5 \% \mathrm{CO}_{2}$ incubator at $37^{\circ} \mathrm{C}$ for $6 \mathrm{~h}$. Subsequently, the medium was replaced with fresh DMEM containing $10 \%$ FBS and cells were cultured for 48-72 h. A total of $2 \times 10^{5}$ cells that were transfected with miRNAs were incubated for $24 \mathrm{~h}$ at $37^{\circ} \mathrm{C}$, and then in the presence or absence of $15 \mu \mathrm{M}$ of the Akt inhibitor LY294002 (Thermo Fisher Scientific, Inc.) for an additional $6 \mathrm{~h}$ at $37^{\circ} \mathrm{C}$.

Bioinformatics analysis. The miRWalk database (http://www .ma.uni-heidelberg.de/apps/zmf/mirwalk/) and Targetscan online software version 3.1 (targetscan.org/vert_71/)) were used to predict potential target genes for miR-96.

Luciferase reporter assays. The 3'-UTR sequence of ARID2 that was predicted to interact with miR-96, or the mutated sequence within the predicted target sites, was synthesized and inserted into the pGL3 control vector (Promega Corporation, Madison, WI, USA). These constructs were referred to as wild-type or mutant ARID2-3'-UTR, respectively. A total of $24 \mathrm{~h}$ prior to transfection, NP cells were seeded in 24-well plates at a density of $2 \times 10^{5}$ cells/well. Cells were co-transfected with $200 \mathrm{ng}$ wild-type ARID2-3'-UTR vector or mutant ARID2-3'-UTR vector along with $500 \mathrm{nM}$ miR-96 mimic, miR-96 inhibitor, or control miRNA and 10 ng pRL-SV40 Renilla plasmid (Promega Corporation) using Lipofectamine 2000. A total of $48 \mathrm{~h}$ post-transfection, cells were harvested and luciferase activity was measured using the Dual-Luciferase Reporter Assay system (Promega Corporation) with a luminometer (Promega Corporation) according to the manufacturer's protocol. Firefly luciferase activity was normalized to Renilla luciferase activity and the 
firefly/Renilla ratio was reported. Results were obtained from three independent experiments.

Reverse transcription-quantitative polymerase chain reaction $(R T-q P C R)$. Total RNA was extracted from $2 \times 10^{5} \mathrm{NP}$ cells using TRIzol reagent (Invitrogen; Thermo Fisher Scientific, Inc.), according to the manufacturer's protocol. Total RNA was reverse transcribed into cDNA using the RevertAid RT Reverse Transcription kit (Thermo Fisher Scientific, Inc.) according to the manufacturer's protocol. The temperature protocol was as follows: Incubation at $37^{\circ} \mathrm{C}$ for $60 \mathrm{~min}$, followed by enzymatic inactivation by incubation at $85^{\circ} \mathrm{C}$ for 5 min. qPCR was performed on cDNA using the Primescript RT-PCR kit (Takara Bio, Inc., Otsu, Japan) on a LightCycler 480 instrument (Roche Diagnostics, Basel, Switzerland). GAPDH was used as a housekeeping gene for ARID2 mRNA expression. miRNA was extracted from $2 \times 10^{5} \mathrm{NP}$ cells using the mirVana miRNA Isolation kit (Thermo Fisher Scientific, Inc.) according to the manufacturer's protocol. miR-96 expression was determined using the miScript SYBR-Green PCR kit (Qiagen, Inc., Valencia, CA, USA) on a LightCycler 480 instrument. U6 was used as a housekeeping gene for miR-96 expression. Thermocycling conditions were as follows: Initial denaturation at $95^{\circ} \mathrm{C}$ for $3 \mathrm{~min}$, followed by 40 cycles of denaturation at $95^{\circ} \mathrm{C}$ for $15 \mathrm{sec}$ and annealing/elongation for $34 \mathrm{sec}$ at $60^{\circ} \mathrm{C}$. The specific primers used were as follows: ARID2, 5'-AGCTCTTGGCAGCTAATCGT-3' (forward), 5'-ACAGGG TCCAGTAAAGCTCAG-3' (reverse); GAPDH 5'-CTGGGC TACACTGAGCACC-3' (forward), 5'-AAGTGGTCGTTG AGGGCAATG-3' (reverse); miR-96, 5'-CAUCUGAAUAGA CGATTAG-3' (forward), 5'-GUAAGCACUAUAAGCCGC-3' (reverse); and U6, 5'-CCCACTCAACAGCGTGTCTC-3' and 5'-CGTCGATTATCTGAATTTGGCCT-3' (reverse). Primers were designed by Applied Biosystems; Thermo Fisher Scientific, Inc. Relative mRNA and miRNA expression was quantified according to the relative $\mathrm{Cq}$ method (26). Experiments were performed in triplicate.

Western blotting. Cells were lysed using radioimmunoprecipitation assay lysis buffer (Beyotime Institute of Biotechnology, Shanghai, China), followed by $5-10 \mathrm{~min}$ boiling and centrifugation at $12,000 \times \mathrm{g}$ for $15 \mathrm{~min}$ at $4^{\circ} \mathrm{C}$ to obtain the protein supernatants. Protein concentration was quantified using a bicinchoninic acid protein assay kit (Pierce; Thermo Fisher Scientific, Inc.). Equal amounts of extracted protein samples $(50 \mu \mathrm{g})$ were separated by $10 \%$ SDS-PAGE (Beyotime Institute of Biotechnology) and transferred onto polyvinylidene difluoride membranes (Thermo Fisher Scientific, Inc.), which were then blocked with 5\% non-fat dried milk in PBS containing $0.1 \%$ Tween-20 (PBST; Beyotime Institute of Biotechnology) overnight at $4^{\circ} \mathrm{C}$. The membrane was then incubated with the following primary antibodies at room temperature for $3 \mathrm{~h}$ : Anti-ARID2 (cat no. 13594; 1:100; Cell Signaling Technology, Inc., Danvers, MA, USA), anti-Akt (cat no. 4691; 1:100; Cell Signaling Technology, Inc.), anti-phosphorylated (phospho) 473-Akt (cat no. 4060; 1:100; Cell Signaling Technology, Inc.), anti-cyclin D1 (cat no. 2978; 1:100; Cell Signaling Technology, Inc.) and anti-GAPDH (cat no. ab9485; 1:100; Abcam, Cambridge, UK). Following washing with PBST 3 times, the membrane was incubated with goat anti-rabbit horseradish peroxidase-conjugated secondary antibody (cat no. ab7090; 1:5,000; Abcam) for $40 \mathrm{~min}$ at room temperature. Protein bands were visualized using an enhanced chemiluminescence kit (GE Healthcare Life Sciences, Little Chalfont, UK). Blots were semi-quantified by densitometry using Image-Pro Plus software version 6.0 (Media Cybernetics, Inc., Rockville, MD, USA) and normalized to GAPDH.

Cell proliferation. NP cells were seeded into 96-well plates at a density of $1 \times 10^{4}$ cells/well and cultured at $37^{\circ} \mathrm{C}$ for $24 \mathrm{~h}$ after transfection. Cell viability was measured every $24 \mathrm{~h}$ for 3 days by adding $10 \%$ Cell Counting Kit (CCK)-8 reagent (Dojindo Molecular Technologies, Inc., Kumamoto, Japan). After incubation at room temperature for $10 \mathrm{~min}$, the absorbance at $450 \mathrm{~nm}$ was measured using a spectrophotometer. Cell proliferation was estimated using predefined absorbance values. All experiments were performed in triplicate.

Statistical analysis. Data are presented as the mean \pm standard deviation of 3 independent experiments. Statistical analysis was performed using SPSS statistical software version 17.0 (SPSS, Inc., Chicago, IL, USA). The statistical significance of the differences between groups was assessed using Student's t-test for air-wise comparisons or one-way analysis of variance followed by a post hoc Bonferoni or Fisher's exact test for multiple comparisons. The correlation between the expression of miR-96 and ARID2 mRNA was estimated using Spearman's correlation analysis. $\mathrm{P}<0.05$ was considered to indicate a statistically significant difference.

\section{Results}

miR-96 expression is increased in patients with IDD. In order to understand the role of miR-96 in the development of IDD, the expression of miR-96 in human degenerated NP tissues and normal controls was investigated. The expression of miR-96 in patients with IDD was significantly higher compared with healthy controls $(\mathrm{P}<0.01$; Fig. 1A). miR-96 expression was increased progressively in patients with Pfirrmann grades III, IV and V disc degeneration, while the expression of ARID2 was decreased as the grade increased. $(\mathrm{P}<0.05$; Fig. 1B). In patients with IDD, the expression of ARID2 was significantly decreased compared with healthy controls $(\mathrm{P}<0.01$; Fig. $1 \mathrm{C})$. Correlation analysis demonstrated that there was a negative correlation between miR-96 expression and ARID2 mRNA levels in patients with IDD $(r=-0.8681 ; \mathrm{P}<0.01$; Fig. 1D).

miR-96 induces NP cell proliferation. As miR-96 expression was demonstrated to be associated with the grade of disc degeneration in patients, the effects of miR-96 expression on NP cell proliferation were subsequently investigated. NP cells were transfected with an miR-96 mimic or miR-96 inhibitor. As determined by RT-qPCR, the miR-96 mimic significantly increased the level of miR-96 in NP cells, while the miR-96 inhibitor significantly decreased its expression $(\mathrm{P}<0.001$; Fig. 2A and B). Furthermore, the CCK-8 assay demonstrated that overexpression of miR-96 increased NP cell proliferation compared with miR-control-transfected cells (Fig. 2C). By contrast, downregulation of miR-96 
A

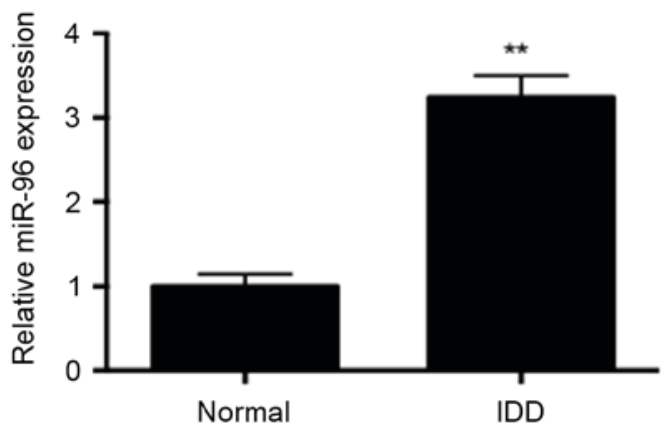

C

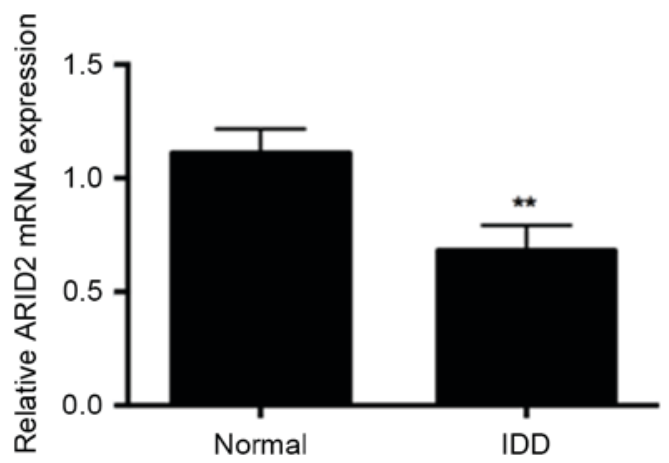

B

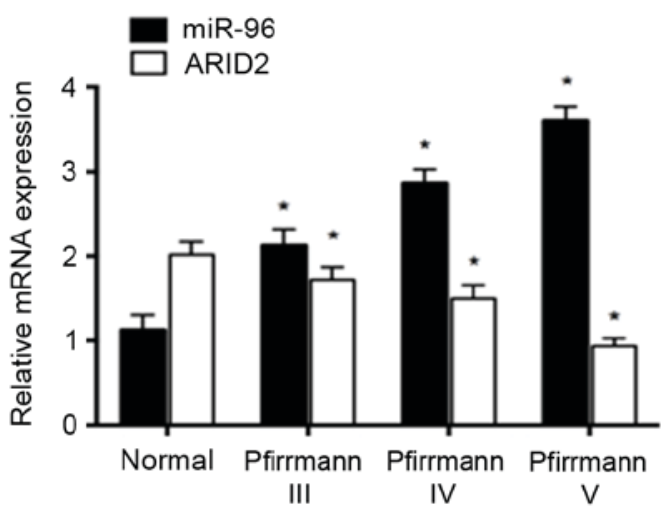

D

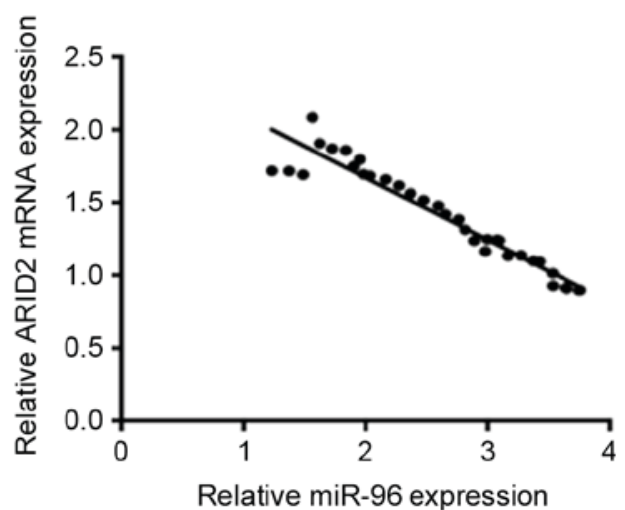

Figure 1. The expression of miR-96 and ARID2 mRNA in human NP tissues. (A) miR-96 expression in NP tissues from patients with IDD and normal controls. The degenerated NP tissues exhibited high expression of miR-96 compared with the control $(n=4)$. (B) miR-96 and ARID2 mRNA expression in NP tissues from healthy controls and patients with IDD at Pfirrmann stages III, IV and V ( $\mathrm{n}=4-6)$. (C) ARID2 mRNA expression in NP tissues from patients with IDD and normal controls $(\mathrm{n}=4)$. (D) A statistically significant inverse correlation was observed between miR-96 and ARID2 mRNA levels in NP tissues, as determined by Spearman's correlation analysis $(\mathrm{n}=30)$. Data are presented as the mean \pm standard deviation. ${ }^{~} \mathrm{P}<0.05$ and ${ }^{* *} \mathrm{P}<0.01$ vs. normal group. miR, microRNA; ARID2, AT-rich interaction domain 2; NP, nucleus pulposus; IDD, intervertebral disc degeneration.

A

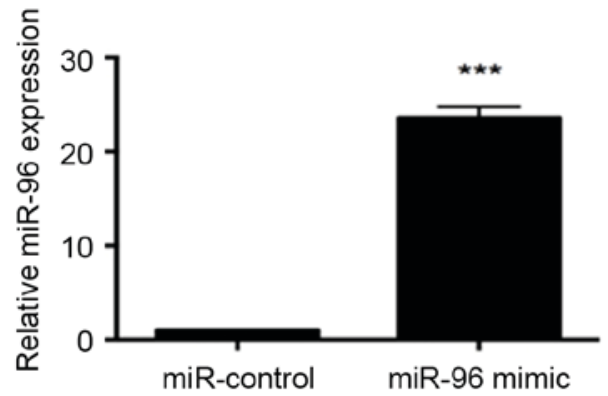

C

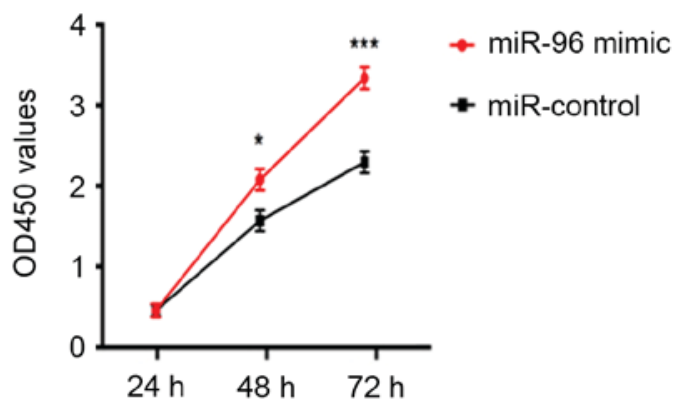

B

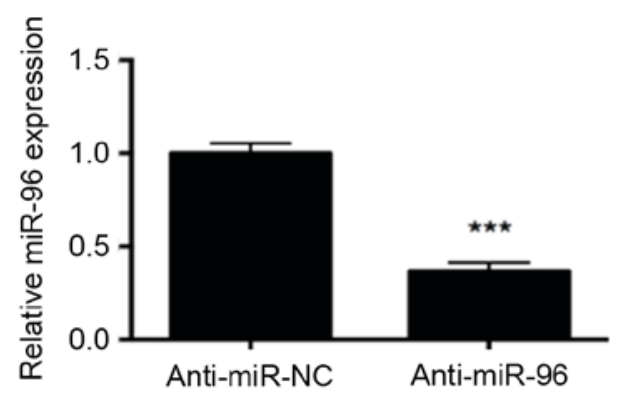

D

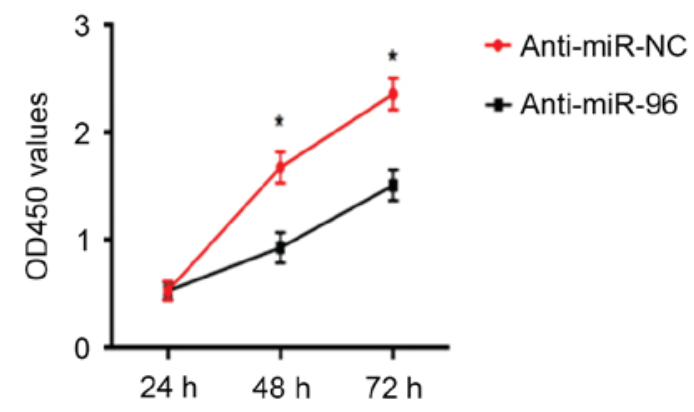

Figure 2. Overexpression of miR-96 promotes human NP cell proliferation. miR-96 expression was measured by reverse transcription-quantitative polymerase chain reaction. Cells were transfected with (A) miR-96 mimic or miR-control and (B) anti-miR-96 or anti-miR-NC ( $\mathrm{n}=3$ ). Cell proliferation, measured by the Cell Counting Kit-8 assay at 24, 48 and $72 \mathrm{~h}$ after transfection, was (C) increased by overexpressing miR-96 in NP cells and (D) inhibited by inhibition of miR-96 $(\mathrm{n}=4)$. Data are presented as the mean \pm standard deviation. ${ }^{*} \mathrm{P}<0.05$ and ${ }^{* * * *} \mathrm{P}<0.001$ vs. miR-control or anti-miR-NC at same time point. miR, microRNA; NP, nucleus pulposus; NC, negative control; OD, optical density. 
A

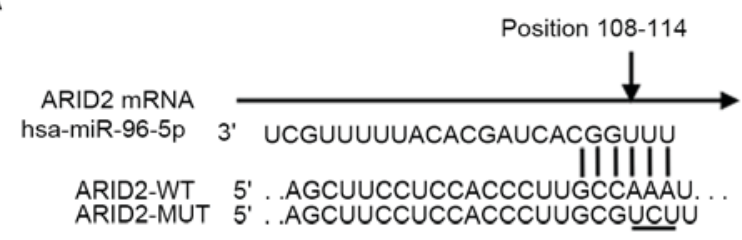

C

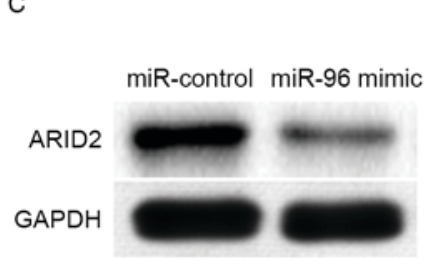

D

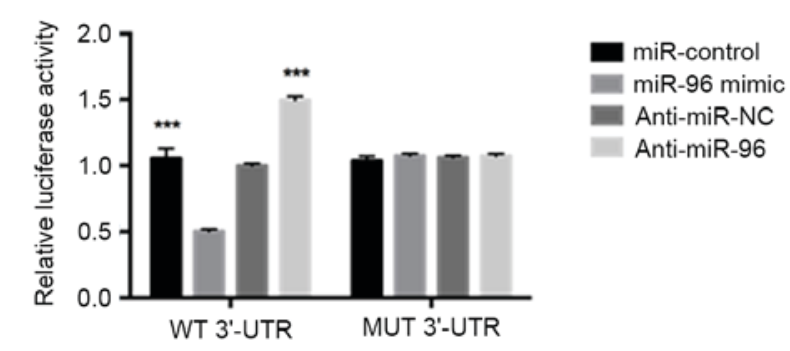

B
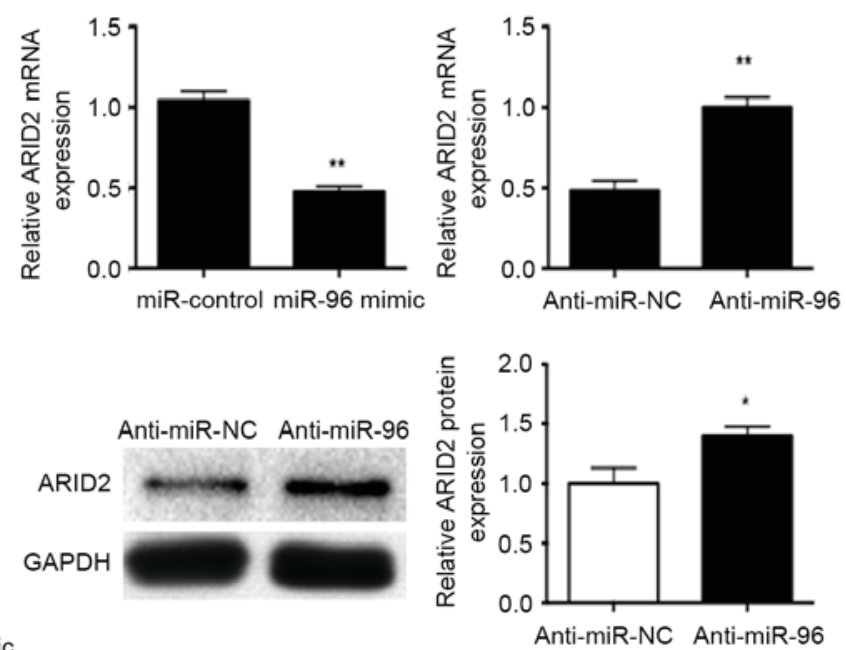

Figure 3. ARID2 is a direct target of miR-96 in human NP cells. (A) TargetScan software indicated that the 3'-UTR of ARID2 contained highly conserved putative miR-96 binding sites. (B) ARID2 mRNA expression in NP cells transfected with the miR-96 mimic or miR-control, and anti-miR-96 or anti-miRNA-NC $(n=3)$. (C) miR-96 mimic transfection reduced the expression of ARID2 protein and inhibition of miR-96 increased the level of ARID2 protein in NP cells $(n=3)$. (D) miR-96 mimic significantly suppressed luciferase activity in NP cells that carried the WT 3'-UTR of ARID2 but not in NP cells that carried the MUT 3'-UTR of ARID2. Anti-miR-96 led to a significant increase in the luciferase activity of NP cells carrying the WT 3'-UTR of ARID2 (n=4). Data are presented as the mean + standard deviation. ${ }^{*} \mathrm{P}<0.05,{ }^{* *} \mathrm{P}<0.01$ and ${ }^{* * *} \mathrm{P}<0.001$ vs. miR-control or anti-miR-NC. ARID2, AT-rich interaction domain 2 ; miR, microRNA; NP, nucleus pulposus; UTR, untranslated region; NC, negative control; WT, wild-type; MUT, mutant.

significantly reduced the proliferation of NP cells compared with cells transfected with anti-miR-NC ( $<<0.05$; Fig. 2D). These results indicate that miR-96 promoted the proliferation of NP cells.

ARID2 is a direct target of miR-96 in IDD. To investigate the mechanism of miR-96 regulation in IDD, bioinformatics analysis was performed using miRWalk and TargetScan to predict the putative target genes of miR-96. Among the target genes identified, the binding sites for miR-96 in the 3'-UTR of ARID2 were conserved across species (Fig. 3A). To confirm the regulatory role of miR-96 on ARID2, RT-qPCR and western blotting were performed to determine the effect of miR-96 on ARID2 mRNA and protein levels, respectively. miR-96 mimic significantly decreased, while inhibition of miR-96 significantly increased, ARID2 mRNA ( $\mathrm{P}<0.01$; Fig. 3B) and protein $(\mathrm{P}<0.05$; Fig. $3 \mathrm{C})$ levels, compared with the respective controls. In addition, miR-96 mimic inhibited the luciferase reporter activity of ARID2 containing a wild-type 3'-UTR, but did not suppress the activity of ARID2 with a mutant 3'-UTR. Suppression of miR-96 by anti-miR-96 increased the luciferase reporter activity of wild-type ARID2 3'-UTR (P<0.01; Fig. 3D). However, with the mutant ARID2 3'-UTR constructs, no relative increase in reporter activity was observed (Fig. 3D). These results indicate that ARID2 is a direct target gene for miR-96 and that miR-96 downregulates ARID2 expression.
miR-96 promotes cell proliferation by activating Akt phosphorylation. Previous studies have demonstrated that activation of Akt signaling by phosphorylation has an important role in cell proliferation $(27,28)$. Therefore, the presents study investigated the molecular mechanism underlying the miR-96-mediated promotion of NP cell proliferation. Overexpression of miR-96 using miR-96 mimic led to significantly increased Akt phosphorylation in NP cells $(\mathrm{P}<0.05$; Fig. 4A), while inhibition of miR-96 significantly decreased Akt phosphorylation $(\mathrm{P}<0.05$; Fig. 4B), compared with the respective controls. Additionally, the expression of cyclin D1, a downstream effector of Akt signaling and a key regulator of cell cycle progression and proliferation, was increased by miR-96 mimic and decreased by miR-96 inhibitor treatment in NP cells $(\mathrm{P}<0.05$; Fig. 4A and B).

ARID2 is involved in the effect of miR-96 on NP cell proliferation. To confirm that ARID2 was a functional target of miR-96, the present study used an ARID2 expression plasmid to significantly increase ARID2 protein expression in NP cells, compared with NP cells treated with control vector $(\mathrm{P}<0.001$; Fig. 5A). In addition, the results demonstrated that overexpression of ARID2 significantly inhibited Akt phosphorylation in NP cells, compared with control vector-transfected cells $(\mathrm{P}<0.05$; Fig. 5A). Furthermore, overexpression of ARID2 in NP cells, and treatment with the 
A

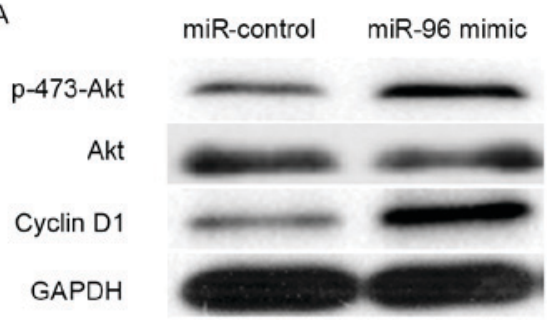

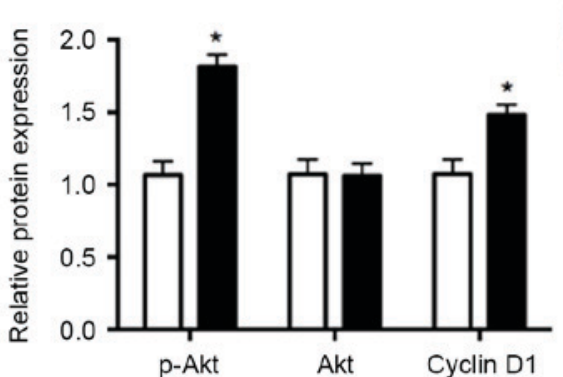

B

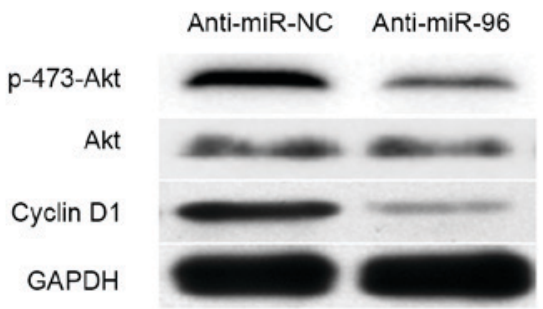

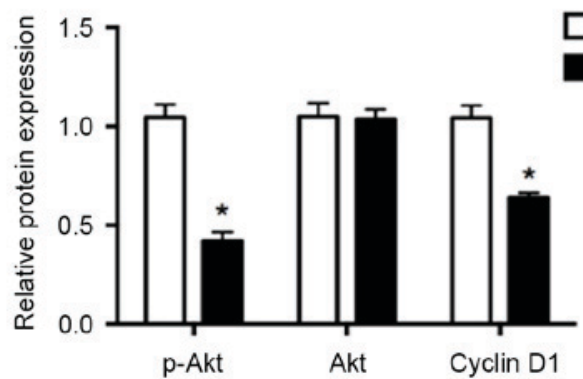

Figure 4. Akt phosphorylation is essential for miR-96-induced human NP cell proliferation. (A and B) Representative western blots and densitometric analysis results for p-Akt, total Akt and cyclin D1 protein levels in NP cells transfected with (A) miR-96 mimic or miR-control and (B) anti-miR-96 or anti-miR-NC $(\mathrm{n}=3)$. Data are presented as the mean \pm standard deviation. ${ }^{*} \mathrm{P}<0.05$ vs. miR-control or anti-miR-NC. miR, microRNA; NP, nucleus pulposus; p-, phosphorylated-; NC, negative control.
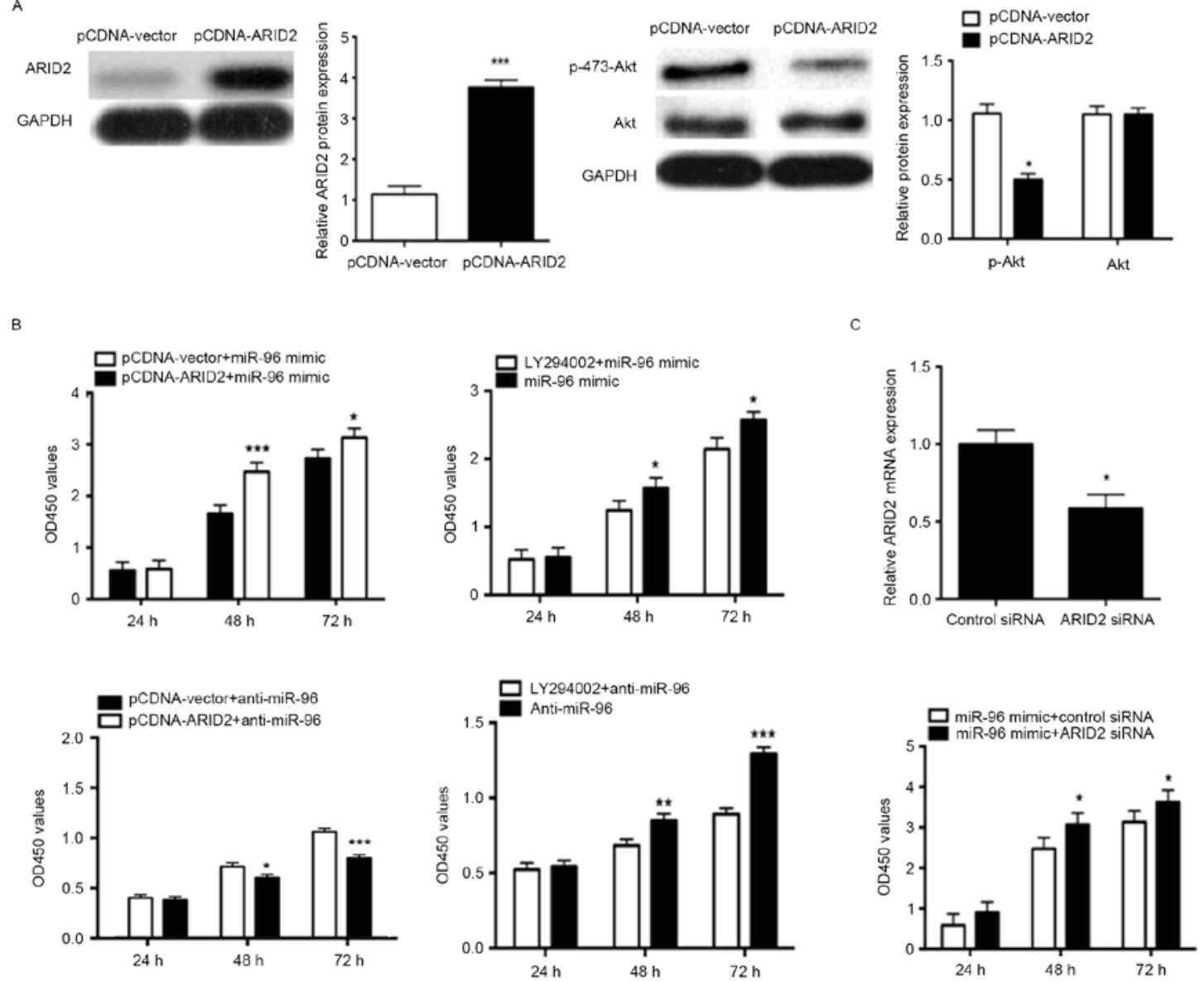

Figure 5. ARID2 is involved in miR-96-induced human NP cell proliferation. (A) NP cells were transfected with the ARID2 expression plasmid and subjected to western blot analysis for ARID2, p-Akt and total Akt expression (n=3). (B) Proliferative effects of miR-96 in NP cells were blocked by overexpression of ARID2 or treatment with the Akt inhibitor LY294002 (n=3). (C) Proliferative effects of miR-96 in NP cells were promoted by ARID2 siRNA ( $=3$ ). Data are presented as the mean \pm standard deviation. ${ }^{*} \mathrm{P}<0.05,{ }^{* *} \mathrm{P}<0.01$ and ${ }^{* * * *} \mathrm{P}<0.001$ vs. respective control group at same time point. ARID2, AT-rich interaction domain 2; miR, microRNA; NP, nucleus pulposus; p-, phosphorylated-; siRNA, small interfering RNA. 
Akt inhibitor LY294002, decreased the proliferative effect of miR-96, while ARID2 overexpression and Akt inhibitor LY294002 enhanced the antiproliferative effect of miR-96 downregulation $(\mathrm{P}<0.05$; Fig. $5 \mathrm{~B})$. In order to further confirm that ARID2 mediates the proliferation effects of miR-96, ARID2 was knocked down using siRNA, and the results demonstrated that ARID2 siRNA increased the miR-96 mimic-induced proliferation of NP cells $(\mathrm{P}<0.05$; Fig. $5 \mathrm{C})$.

\section{Discussion}

miRNAs are key modulators of a variety of biological and pathological processes, which include cell growth, differentiation, apoptosis and carcinogenesis, through the direct regulation of gene expression. However, the molecular mechanisms of miRNAs in disc degeneration remain largely unknown. In the present study, the expression of miR-96 was significantly increased in degenerated NP tissues compared with control NP tissues, and positively associated with the disc degeneration grade. These results indicate that miR-96 may have a critical role in IDD. Furthermore, the results demonstrated that overexpression of miR-96 increased the proliferation of NP cells, and ARID2 was identified as a direct novel target of miR-96. In addition, ARID2 mRNA expression was downregulated in degenerated NP tissues compared with control NP tissues, and ARID2 mRNA expression was inversely correlated with the expression of miR-96. Mechanistically, the present study demonstrated that overexpression of miR-96, using an miR-96 mimic, activated Akt signaling by directly targeting ARID2. Furthermore, the proliferative effect of miR-96 was decreased by overexpressing ARID2. These results indicated that miR-96, and the downstream ARID2/Akt pathway, may serve as potential novel therapeutic targets in the treatment of IDD.

Previous reports have demonstrated that miR-96 expression is frequently increased in various types of human cancer, and is involved in regulating various developmental and cellular processes, including cell proliferation, apoptosis, migration and invasion $(29,30)$. In addition, upregulation of miR-96 was reported to be closely associated with the progression of various tumor types and their pathological grade, including lung, esophageal, hepatocellular and breast cancers (30-33). However, the role of miR-96 in degenerated NP tissues, and its importance in the pathogenesis of IDD, are yet to be established. To investigate the mechanisms underlying the role of miR-96 in the development of IDD, the present study transfected NP cells with miR-96 mimic in order to overexpress miR-96. Overexpression of miR-96 led to a significant increase in NP cell proliferation. Previous studies have reported that the formation of NP cell clusters and the proliferation of fibrocartilaginous tissue have important roles in the development of IDD (34-36). The results of the current study indicated that increased NP cell proliferation, which was promoted by miR-96 upregulation, may be a potential mechanism in the development of IDD.

ARID2, a novel tumor suppressor gene, was initially identified in the polybromo-associated BRG1-associated factor complex, which is a SWI/SNF chromatin-remodeling complex that functions in ligand-dependent activation of transcription by nuclear receptors (37). Previous studies have demonstrated that ARID2 is involved in various biological processes and suppression of ARID2 promoted cell proliferation by inducing G1/S transition in hepatoma cells $(38,39)$. Furthermore, a number of studies have reported that ARID2 expression is downregulated in certain cancer types, including hepatocellular carcinoma and gastric cancer $(39,40)$. In the current study, miR-96 negatively regulated ARID2 mRNA and protein levels in NP cells. In addition, the vector expressing the mutant form of the ARID2 3'-UTR was resistant to miR-96 inhibition in NP cells. Therefore, the results indicate that ARID2 is a direct target of miR-96.

Akt phosphorylation promotes cell cycle progression and proliferation via cyclin D1 upregulation. Loss of ARID2 is reported to enhance Akt activation, upregulation of cyclin D1 and downregulation of p27, which subsequently increases growth and survival in various cell types (39-41). In the present study, overexpression of miR-96 increased Akt activation in NP cells. Furthermore, the effect of altered miR-96 expression on the proliferation of NP cells was reduced by overexpressing ARID2 or by the Akt inhibitor LY294002. These results indicate that miR-96 may promote the proliferation of NP cells by directly targeting the ARID2/Akt signaling pathway.

In conclusion, the results of the present study demonstrated that miR-96 is upregulated in human degenerated NP tissues and its expression level is positively associated with the disc degeneration grade. Overexpression of miR-96 promoted NP cell proliferation by targeting ARID2 to activate Akt signaling. Combined, these results demonstrate an important role for miR-96 and the downstream ARID2/Akt pathway in the pathogenesis of IDD. Importantly, these results have identified a potential novel therapeutic target for the treatment of IDD.

\section{Acknowledgements}

The authors would like to thank members of the laboratory of Dr. Yu (Y Department of Institute for Nutritional Sciences, Shanghai Institutes for Biological Sciences, Chinese Academy of Sciences) for technical advice and critical reviews of the manuscript. The present study was supported by the National Natural Science Foundation of P.R. China (grant nos. 31260233 and 81460198).

\section{References}

1. Feuerstein M, Marcus SC and Huang GD: National trends in nonoperative care for nonspecific back pain. Spine J 4: 56-63, 2004.

2. Katz JN: Lumbar disc disorders and low-back pain: Socioeconomic factors and consequences. J Bone Joint Surg Am 88 (Suppl 2): S21-S24, 2006.

3. Lee HJ, Seo JC, Kwak MA, Park SH, Min BM, Cho MS, Shin I, Jung JY and Roh WS: Acupuncture for low back pain due to spondylolisthesis: Study protocol for a randomized controlled pilot trial. Trials 15: 105, 2014.

4. Freemont TJ, LeMaitre C, Watkins A and Hoyland JA: Degeneration of intervertebral discs: Current understanding of cellular and molecular events, and implications for novel therapies. Expert Rev Mol Med 2001: 1-10, 2001.

5. Mooney V: The classification of low back pain. Ann Med 21: 321-325, 1989.

6. Li Z, Liang J, Wu WK, Yu X, Yu J, Weng X and Shen J: Leptin activates RhoA/ROCK pathway to induce cytoskeleton remodeling in nucleus pulposus cells. Int J Mol Sci 15: 1176-1188, 2014.

7. Liu G, Cao P, Chen H, Yuan W, Wang J and Tang X: miR-27a regulates apoptosis in nucleus pulposus cells by targeting PI3K. PLoS One 8: e75251, 2013. 
8. Li Z, Shen J, Wu WK, Yu X, Liang J, Qiu G and Liu J: Leptin induces cyclin D1 expression and proliferation of human nucleus pulposus cells via JAK/STAT, PI3K/Akt and MEK/ERK pathways. PLoS One 7: e53176, 2012.

9. Johnson WE, Eisenstein SM and Roberts S: Cell cluster formation in degenerate lumbar intervertebral discs is associated with increased disc cell proliferation. Connect Tissue Res 42: 197-207, 2001.

10. Liu H, Huang X, Liu X, Xiao S, Zhang Y, Xiang T, Shen X, Wang $\mathrm{G}$ and Sheng B: miR-21 promotes human nucleus pulposus cell proliferation through PTEN/AKT signaling. Int J Mol Sci 15: 4007-4018, 2014.

11. Brennecke J and Cohen SM: Towards a complete description of the microRNA complement of animal genomes. Genome Biol 4 $228,2003$.

12. Ambros V: The functions of animal microRNAs. Nature 431: $350-355,2004$

13. Soifer HS, Rossi JJ and Saetrom P: microRNAs in disease and potential therapeutic applications. Mol Ther 15: 2070-2079, 2007.

14. Kloosterman WP and Plasterk RH: The diverse functions of microRNAs in animal development and disease. Dev Cell 11: 441-450, 2006

15. Yu X and Li Z: microRNAs regulate vascular smooth muscle cell functions in atherosclerosis (review). Int J Mol Med 34: 923-933, 2014.

16. Lu J, Getz G, Miska EA, Alvarez-Saavedra E, Lamb J, Peck D, Sweet-Cordero A, Ebert BL, Mak RH, Ferrando AA, et al microRNA expression profiles classify human cancers. Nature 435: 834-838, 2005

17. Volinia S, Calin GA, Liu CG, Ambs S, Cimmino A, Petrocca F, Visone R, Iorio M, Roldo C, Ferracin M, et al: A microRNA expression signature of human solid tumors defines cancer gene targets. Proc Natl Acad Sci USA 103: 2257-2261, 2006.

18. Li W, Wang P, Zhang Z, Wang W, Liu Y and Qi Q: miR-184 regulates proliferation in nucleus pulposus cells by targeting GAS1. World Neurosurg 97: 710-715.e1, 2017.

19. Chen B, Huang SG, Ju L, Li M, Nie FF, Zhang Y, Zhang YH, Chen X and Gao F: Effect of microRNA-21 on the proliferation of human degenerated nucleus pulposus by targeting programmed cell death 4. Braz J Med Biol 49: pii, 2016.

20. Jing W and Jiang W: microRNA-93 regulates collagen loss by targeting MMP3 in human nucleus pulposus cells. Cell Prolif 48 284-292, 2015

21. Yamada Y, Enokida H, Kojima S, Kawakami K, Chiyomaru T, Tatarano S, Yoshino H, Kawahara K, Nishiyama K, Seki N and Nakagawa M: miR-96 and miR-183 detection in urine serve as potential tumor markers of urothelial carcinoma: Correlation with stage and grade, and comparison with urinary cytology. Cancer Sci 102: 522-529, 2011

22. Xu XM, Qian JC, Deng ZL, Cai Z, Tang T, Wang P, Zhang KH and Cai JP: Expression of miR-21, miR-31, miR-96 and miR-135b is correlated with the clinical parameters of colorectal cancer. Oncol Lett 4: 339-345, 2012.

23. Liu Y, Han Y, Zhang H, Nie L, Jiang Z, Fa P, Gui Y and Cai Z: Synthetic miRNA-mowers targeting miR-183-96-182 cluster or miR-210 inhibit growth and migration and induce apoptosis in bladder cancer cells. PLoS One 7: e52280, 2012.

24. Pfirrmann CW, Metzdorf A, Zanetti M, Hodler J and Boos N: Magnetic resonance classification of lumbar intervertebral disc degeneration. Spine (Phila Pa 1976) 26: 1873-1878, 2001.
25. Li Z, Shen J, Wu WK, Yu X, Liang J, Qiu G and Liu J: The role of leptin on the organization and expression of cytoskeleton elements in nucleus pulposus cells. J Orthop Res 31: 847-857, 2013.

26. Livak KJ and Schmittgen TD: Analysis of relative gene expression data using real-time quantitative PCR and the 2(-Delta Delta C(T)) method. Methods 25: 402-528, 2001.

27. Liu X, Liao W, Yuan Q, Ou Y and Huang J: TTK activates Akt and promotes proliferation and migration of hepatocellular carcinoma cells. Oncotarget 6: 34309-34320, 2015.

28. Wang ZQ, Cai Q, Hu L, He CY, Li JF, Quan ZW, Liu BY, $\mathrm{Li} \mathrm{C}$ and Zhu ZG: Long noncoding RNA UCA1 induced by SP1 promotes cell proliferation via recruiting EZH2 and activating AKT pathway in gastric cancer. Cell Death Dis 8: e2839, 2017.

29. Li J, Li P, Chen T, Gao G, Chen X, Du Y, Zhang R, Yang R, Zhao W, Dun S, et al: Expression of microRNA-96 and its potential functions by targeting FOXO3 in non-small cell lung cancer. Tumour Biol 36: 685-692, 2015.

30. Zhang J, Kong X, Li J, Luo Q, Li X, Shen L, Chen L and Fang L: miR-96 promotes tumor proliferation and invasion by targeting RECK in breast cancer. Oncol Rep 31: 1357-1363, 2014.

31. Wu L, Pu X, Wang Q, Cao J, Xu F, Xu LI and Li K: miR-96 induces cisplatin chemoresistance in non-small cell lung cancer cells by downregulating SAMD9. Oncol Lett 11: 945-952, 2016.

32. Xia H, Chen S, Chen K, Huang $\mathrm{H}$ and $\mathrm{Ma} \mathrm{H}$ : miR-96 promotes proliferation and chemo-or radioresistance by down-regulating RECK in esophageal cancer. Biomed Pharmacother 68: 951-958, 2014.

33. Xu D, He X, Chang Y, Xu C, Jiang X, Sun S and Lin J: Inhibition of miR-96 expression reduces cell proliferation and clonogenicity of HepG2 hepatoma cells. Oncol Rep 29: 653-661, 2013.

34. Cai P, Yang T, Jiang X, Zheng M, Xu G and Xia J: Role of miR-15a in intervertebral disc degeneration through targeting MAP3K9. Biomed Pharmacother 87: 568-574, 2017.

35. Wang $X, L v$ G, Li J, Wang B, Zheng $Q$ and Lu C: LncRNA-RP11-296A18.3/miR-138/HIF1A pathway regulates the proliferation ECM synthesis of human nucleus pulposus cells (HNPCs). J Cell Biochem: May 24, 2017 doi: 10.1002/jcb.26166. (Epub ahead of print)

36. Li Z, Yu X, Shen J, Chan MT and Wu WK: microRNA in intervertebral disc degeneration. Cell Prolif 48: 278-283, 2015.

37. Madden HP, Breslin RJ, Wasserkrug HL, Efron G and Barbul A: Stimulation of $\mathrm{T}$ cell immunity by arginine enhances survival in peritonitis. J Surg Res 44: 658-663, 1988.

38. Yu P, Wu D, You Y, Sun J, Lu L, Tan J and Bie P: miR-208-3p promotes hepatocellular carcinoma cell proliferation and invasion through regulating ARID2 expression. Exp Cell Res 336: 232-241, 2015

39. Zhang L, Wang W, Li X, He S, Yao J, Wang X, Zhang D and Sun X: microRNA-155 promotes tumor growth of human hepatocellular carcinoma by targeting ARID2. Int J Oncol 48: 2425-2434, 2016

40. Aso T, Uozaki H, Morita S, Kumagai A and Watanabe M: Loss of ARID1A, ARID1B, and ARID2 expression during progression of gastric cancer. Anticancer Res 35: 6819-6827, 2015.

41. Duan Y, Tian L, Gao Q, Liang L, Zhang W, Yang Y, Zheng Y, Pan E, Li S and Tang N: Chromatin remodeling gene ARID2 targets cyclin D1 and cyclin E1 to suppress hepatoma cell progression. Oncotarget 7: 45863-45875, 2016. 